\title{
Erratum to: An optimal gap theorem
}

\section{Lei Ni}

Published online: 18 October 2013

(C) Springer-Verlag Berlin Heidelberg 2013

\section{Erratum to: Invent math (2012) 189:737-761 DOI 10.1007/s00222-012-0375-6}

Here we shall use the same notations of the original paper. The references to equations, lemmas, propositions, theorems are with respect to the paper unless stated otherwise.

The argument provided for the proof of Proposition 3.1 is not complete. It overlooks the fact that the solution 2-form $\eta(x, t)$ of the problem (3.1) may contain non-trivial $(2,0)$ and $(0,2)$ parts. Hence Proposition 3.1 needs a correction. We thank Gilles Carron for pointing out this oversight in the original proof of Proposition 3.1 and his comments. We also thank Luen-Fai Tam for helpful related discussions. We refer interested reader to [5] for an alternate proof of Theorem 1.2.

Since the preservation of the positivity for solution 2-forms solving the problem (3.1) in the paper, without knowing that the 2-form being (1,1)-type, seems elusive (even for the $(1,1)$ part projection of the 2 -form solutions) we replace (3.1) with the following initial boundary value problem on $(1,1)$ form $\eta$. Let $\Omega$ be a smooth bounded domain in $M$. For a given point $p \in$ $\partial \Omega$, let $v$ be the unit outward normal in a small neighborhood near $p$. We

The online version of the original article can be found under doi:10.1007/s00222-012-0375-6.

\section{Ni (凶)}

Department of Mathematics, University of California at San Diego, La Jolla, CA 92093, USA

e-mail: lni@math.ucsd.edu 
also extending $v(x)$ by parallel transport along the geodesic $\exp _{x}(t v(x))$, for $|t| \leq \epsilon$ and $x \in \partial \Omega$, to a neighborhood of $\partial \Omega$. For any $p \in \partial \Omega$, pick a small neighborhood of it and choose a unitary frame $\left\{e_{i}\right\}, 1 \leq i \leq m$, so that $e_{m}=$ $\frac{1}{2}(v-\sqrt{-1} J v)$. Let $\left\{e_{i}^{*}\right\}$ be the dual. For $\eta=\sqrt{-1} \sum_{1 \leq i, j \leq m} \eta_{i} e_{i}^{*} \wedge \overline{e_{j}^{*}}$ we define a boundary operator $\mathcal{N}(\eta)$ by

$$
\mathcal{N}(\eta)=\sqrt{-1} \sum_{1 \leq i, j \leq m-1} \nabla_{\nu} \eta_{i} \bar{j}_{i}^{*} \wedge \overline{e_{j}^{*}}
$$

One can check that this is well-defined. Now we replace (3.1) with the following initial boundary value problem:

$$
\left\{\begin{array}{l}
\left(\frac{\partial}{\partial t}+\Delta_{\bar{\partial}}\right) \eta_{\mu}(x, t)=0, \quad \text { on } \Omega_{\mu} \times[0, \infty), \\
\iota_{\nu} \eta_{\mu}=0, \quad \mathcal{N}\left(\eta_{\mu}\right)=0, \quad \text { on } \partial \Omega_{\mu} \times[0, \infty), \\
\eta_{\mu}(x, 0)=\operatorname{Ric}(x), \quad \text { on } \Omega_{\mu} .
\end{array}\right.
$$

Here $\iota_{\nu} \eta$ (which is the same as $\mathbf{n} \eta$ in the paper) denotes the contraction with the unit exterior normal vector $v$. It can be checked that this is a parabolic initial boundary value problem. In particular, the total number of the boundary conditions matches with the number of unknowns (unlike the problem (3.1) restricted within the space of $(1,1)$-forms).

Proposition 0.1 Assume that $\Omega$ is a smooth bounded domain. Let $\eta(x, t)$ be the unique solution of the initial-boundary value problem $(0.1)$. Then $\eta(x, t)$ is a real nonnegative $(1,1)$-form provided that $\eta(x, 0)$ is real nonnegative.

Proof With the above local frame, the boundary conditions amount to $\eta_{i \bar{m}}=$ $\eta_{m \bar{i}}=\eta_{m \bar{m}}=0$ and $\nabla_{\nu} \eta_{i} \bar{j}=0$ for $1 \leq i, j \leq m-1$. The original proof can be carried over verbatim.

Now we have to modify the proof of Theorem 1.2 accordingly since we no longer have the $d$-closedness of the solution. First let $u_{\mu}(x, t)=\Lambda \eta_{\mu}$. The boundary value conditions imply that $\nabla_{\nu}\left(\eta_{\mu}\right)_{i \bar{i}}=0$ for $1 \leq i \leq m-1$ and $\nabla_{\nu}\left(\eta_{\mu}\right)_{m \bar{m}} \leq 0$ due to that $\left(\eta_{\mu}\right)_{m \bar{m}} \geq 0$ and $\left(\eta_{\mu}\right)_{m \bar{m}}=0$ on the boundary. Under the assumption (1.1) of the paper, $u_{\mu}(x, t)$ as a solution to the heat equation, satisfying $\frac{\partial u_{\mu}}{\partial v} \leq 0$. Moreover, by the argument on page 754 of the paper, $\left\{u_{\mu}\right\}$ is bounded over $K \times[0, T]$ by a constant $C(K, n, \mathcal{S}, T)$. Since $u_{\mu}(x, t)$ dominates $\left\|\eta_{\mu}\right\|$, this shows that problem $(0.1)$ has a long time solution and moreover one can find a convergent subsequence out of $\left\{\eta_{\mu}\right\}$ and obtain, as the limit, a global $(1,1)$-form $\eta(x, t)$, a solution to the Hodge-Laplace heat equation on $M \times[0, \infty)$. Since $\Lambda \eta(x, t)$ solves the heat equation and is positive, the uniqueness theorem implies that $\Lambda \eta(x, t)=u(x, t)$. 
To prove Theorem 1.2, we also need that $u(x, t)$ satisfies the Li-YauHamilton type estimate, in particular, $\frac{\partial}{\partial t}(t u(x, t)) \geq 0$. Now we apply Theorem 1.1 of [2], which after taking $V=0$ asserts, via the Kähler identities, that

$$
u_{t}+\frac{1}{2}\left(\bar{\partial}^{*} \Lambda \bar{\partial}+\partial^{*} \Lambda \partial\right) \eta+\frac{u}{t} \geq 0 .
$$

The proof will be completed if we can establish that $\sigma_{1} \doteqdot \Lambda \bar{\partial} \eta$ and $\sigma_{2} \doteqdot \Lambda \partial \eta$ vanish on $M$.

To prove $\sigma_{1}=0$, we observe that we may also construct $\eta$ as follows. First construct $\eta^{(i)}$, a global $(1,1)$-form solving the Hodge-Laplace heat equation, obtained as the above via exhaustion domains with the initial data $\eta^{(i)}(x, 0)=$ $\phi^{(i)}(x) \operatorname{Ric}(x)$, where $0 \leq \phi^{(i)} \leq 1$ being cut-off functions with support in ball $B_{o}\left(R_{i}\right)$ centered at some fixed point $o \in M$ and $\left|\nabla \phi^{(i)}\right| \leq \frac{C}{R_{i}}$ with $C>0$ independent of $i$ and $R_{i} \rightarrow \infty$. Then obtain $\eta(x, t)$ as the limit of a convergent subsequence of $\left\{\eta^{(i)}(x, t)\right\}$. We shall also denote $\phi^{(i)}(x) \operatorname{Ric}(x)$ by $\rho^{(i)}(x)$. Correspondingly we have $\sigma_{1}^{(i)} \doteqdot \Lambda \bar{\partial} \eta^{(i)}$ and $\sigma_{2}^{(i)} \doteqdot \Lambda \partial \eta^{(i)}$, which converge to $\sigma_{1}$ and $\sigma_{2}$ respectively.

Let $\eta_{\mu}^{(i)}$ be the solution to the initial boundary value problem on $\Omega_{\mu}$. For the boundary problem $(0.1)$ it is easy to see that

$$
\frac{\partial}{\partial \nu}\left\|\eta_{\mu}^{(i)}\right\|^{2}=2\left\langle\nabla_{\nu} \eta_{\mu}^{(i)}, \eta_{\mu}^{(i)}\right\rangle=0
$$

On the other hand, we have the Kodaira-Bochner formula below for any $(1,1)$-form $\eta$

$$
\Delta_{\bar{\partial}} \eta_{i \bar{j}}=-\frac{1}{2} g^{s \bar{t}}\left(\nabla_{\bar{t}} \nabla_{s} \eta_{i \bar{j}}+\nabla_{s} \nabla_{\bar{t}} \eta_{i \bar{j}}\right)+\frac{1}{2}\left(R_{i}^{l} \eta_{l \bar{j}}+R_{\bar{j}}^{\bar{k}} \eta_{i \bar{k}}\right)-R_{i \bar{j}}{ }^{k \bar{l}} \eta_{k \bar{l}} .
$$

Hence it implies that

$$
\begin{aligned}
\left(\frac{\partial}{\partial t}-\Delta\right)\left\|\eta_{\mu}^{(i)}\right\|^{2}= & 2\left\langle\frac{\partial}{\partial t} \eta_{\mu}^{(i)}-\Delta_{\bar{\partial}} \eta_{\mu}^{(i)}, \eta_{\mu}^{(i)}\right\rangle-2\left\|\nabla \eta_{\mu}^{(i)}\right\|^{2} \\
& -g^{p \bar{t}} g^{s \bar{j}}\left(R_{p}^{l}\left(\eta_{\mu}^{(i)}\right)_{l \bar{j}}+R_{\bar{j}}^{\bar{k}}\left(\eta_{\mu}^{(i)}\right)_{p \bar{k}}\right)\left(\eta_{\mu}^{(i)}\right)_{s \bar{t}} \\
& +2 R_{p \bar{j}} \bar{k}^{\bar{l}}\left(\eta_{\mu}^{(i)}\right)_{k \bar{l}}\left(\eta_{\mu}^{(i)}\right)_{s \bar{t}} \\
\leq & -2\left\|\nabla \eta_{\mu}^{(i)}\right\|^{2}
\end{aligned}
$$

This, together with the boundary condition $\frac{\partial}{\partial v}\left\|\eta_{\mu}^{(i)}\right\|^{2}=0$, in particular (as in [3] and [5]) implies that

$$
2 \int_{0}^{t} \int_{\Omega_{\mu}}\left\|\nabla \eta_{\mu}^{(i)}\right\|^{2}(x, s) d \mu d s \leq \int_{\Omega_{\mu}}\left\|\eta_{\mu}^{(i)}\right\|^{2}(x, 0) d \mu .
$$


Letting $\mu \rightarrow \infty$ we obtain that

$$
2 \int_{0}^{t} \int_{M}\left\|\nabla \eta^{(i)}\right\|^{2}(x, s) d \mu d s \leq \int_{M}\left\|\rho^{(i)}\right\|^{2} .
$$

Now observe that $\left\|\sigma_{1}^{(i)}\right\|(x, t)$ is a sub-solution to the heat equation since $\sigma_{1}^{(i)}(x, t)$ is a $(0,1)$-form satisfying the Hodge-Laplace heat equation. Hence by (0.3) the $L^{2}$-maximum principle (see for example Theorem 1.2 of [4]) implies that

$$
\left\|\sigma_{1}^{(i)}\right\|(x, t) \leq \max _{x \in M}\left\|\sigma_{1}^{(i)}\right\|(x, 0) .
$$

Moreover if we let

$$
v^{(i)}(x, t)=\int_{M} H(x, y, t)\left\|\Lambda \bar{\partial}\left(\rho^{(i)}\right)\right\|(y) d \mu(y)
$$

by (0.3) and the $L^{2}$-maximum principle we have that $\left\|\sigma_{1}^{(i)}\right\|(x, t) \leq v^{(i)}(x, t)$. By Lemma 1.1 of [3] we have that

$$
\frac{1}{V_{o}(r)} \int_{B_{o}(r)} v^{(i)}(x, t) d \mu \leq \epsilon(r)
$$

for some $\epsilon(r)$ independent of $i$ with $\epsilon(r) \rightarrow 0$ as $r \rightarrow \infty$. Here we have used assumption (1.1) in the paper and explicit estimates satisfied by $\phi^{(i)}$. Letting $i \rightarrow \infty$ we have that $\left\|\sigma_{1}\right\|(x, t)=0$ by Theorem 1.2 of [1] (applying to $p=1)$. The proof of that $\sigma_{2}=0$ is similar.

This method of getting the monotonicity via the sharp Li-Yau-Hamilton type estimate also works for solution constructed by solving the Dirichlet boundary problems, namely requiring that $\mathbf{t} \eta_{\mu}=0$ and $\mathbf{n} \eta_{\mu}=0$ on the boundary $\partial \Omega_{\mu}$. Another way of proving that $\eta \geq 0$ is to apply Theorem 2.1 of [3] to $\eta^{(i)}$ and show that $\eta^{(i)} \geq 0$.

\section{References}

1. Li, P., Tam, L.-F.: The heat equation and harmonic maps of complete manifolds. Invent. Math. 105(1), 1-46 (1991)

2. Ni, L., Niu, Y.Y.: Sharp differential estimates of Li-Yau-Hamilton type for positive $(p, p)$ forms on Kähler manifolds. Commun. Pure Appl. Math. 64, 920-974 (2011)

3. Ni, L., Tam, L.F.: Plurisubharmonic functions and the structure of complete Kähler manifolds with nonnegative curvature. J. Differ. Geom. 64(3), 457-524 (2003)

4. Ni, L., Tam, L.F.: Kähler-Ricci flow and the Poincaré-Lelong equation. Commun. Anal. Geom. 12(1), 111-141 (2004)

5. Ni, L., Tam, L.F.: Poincaré-Lelong equation via the Hodge Laplace heat equation. Compos. Math. (to appear) 